\section{A Shortage of Privacy Engineers}

Lorrie Faith Cranor and Norman Sadeh | Carnegie Mellon University other users, advertisers, or even the public. ${ }^{2}$ We've heard from managers at some of these companies who are eager to grow privacy expertise on their engineering teams to address these concerns more proactively.

Since the 1990s, Ann Cavoukian, Ontario, Canada's Information and Privacy Commissioner, has been urging companies to adopt $\mathrm{PbD}$, emphasizing the importance of designing products and services with privacy designed in from the beginning rather than patched on later to comply with laws or respond to problems. Although some complain that $\mathrm{PbD}$ can be expensive, Cavoukian argues that if done well, it can be a positive-sum approach in which privacy protections might ultimately improve products or facilitate more cost-effective solutions. On the other hand, when privacy issues aren't addressed early, companies risk costly privacy mistakes that might result in loss of customers, loss of reputation, lawsuits, launch delays, and redesign costs.

$\mathrm{PbD}$ can be applied in software system design as well as in the design of physical systems and business practices. For example, prompted by US healthcare privacy regulation, hospitals and doctors' offices began reconfiguring their waiting areas so that patients couldn't readily overhear conversations about sensitive medical issues. Even relatively small changes, such as moving hospital fax machines from open hallways to locked offices and adding a line behind which pharmacy customers must wait, can improve privacy. However, fax machines in locked offices might no longer be readily accessible to healthcare 
workers who need quick access to faxed information. Offices designed from the beginning to include areas for private conversations and the receipt and storage of private documents can offer better privacy protections as well as an environment conducive to efficient workflows.

Although there are a growing number of guidelines (such as Microsoft's Privacy Guidelines for Developing Software Products and Services $^{3}$ ) and case studies to draw from, there's no simple, universal formula for designing in privacy. $\mathrm{PbD}$ requires engineers with a diverse skill set to understand how both privacy-by-policy and privacyby-architecture mechanisms can interact to protect privacy. Privacyby-policy approaches include privacy notices and mechanisms for obtaining informed consent before data is collected or used. Privacyby-architecture approaches aim to minimize the collection of personal data, anonymize as much of it as feasible, and reduce centralized data storage and processing. ${ }^{4}$ Although organizations can easily implement simple techniques from both approaches, designing meaningful and usable informed consent experiences and implementing robust and sophisticated data minimization or anonymization techniques that preserve essential data utility are both nontrivial tasks.

\section{Shortage of Privacy Engineers}

We've seen an increase in companies recruiting privacy engineers, and many organizations are already reporting a shortage of people who are adequately trained to fill this crucial role. ${ }^{5}$ Recent US Federal Trade Commission settlements with organizations both large and small and pending revisions to privacy laws in places like Europe will fuel increasing demand for privacy professionals. ${ }^{6,7}$

Recently, we've seen announcements recruiting privacy engineers and technical privacy managers at Google, Microsoft, Facebook, Intel, Apple, and the National Institute of Standards and Technology as well as financial companies, privacy-related startups, and government agencies, to name just a few. Already, Google reportedly employs 60 full-time privacy engineers. ${ }^{8}$ Job postings recruit engineers who can "develop technical solutions to help mitigate privacy vulnerabilities"; analyze "software designs and implementations from a privacy and UX perspective"; "research, document, and help remediate design decisions, operating procedures, or processes that may directly or indirectly contribute to future privacy risks"; "create cutting-edge privacy feature prototypes"; "help us lead better on privacy by example"; and "partner with key business, technical, and legal stakeholders across various ... business groups to implement Privacy by Design."

Andrew Swerdlow, a Google privacy analysis engineer, described the role of Google's privacy engineers: "We work closely with legal, policy and other engineers as products are being developed and released. At the beginning of product development, we sit with engineers to help them design products with privacy in mind. During development, we review, audit and test the boundaries of products. After a product launches, we evaluate and reevaluate the product to ensure it remains true to our privacy standards" (www. google.com/about/jobs/lifeat google/meet-andrew-swerdlow -privacy-analysis-engineer.html).

Erin Egan, Facebook's chief privacy officer, explained that Facebook is adding to its team of professionals who develop products with privacy in mind. This team includes privacy engineers and product managers as well as security engineers. "As our team continues to grow, we are looking for candidates who have not only the technical skills to work on our products but a deep understanding of how building privacy into Facebook creates a great experience for our users," Egan told us when we asked her about the skills Facebook was looking for when it hires privacy engineers.

Sidd Stamm, lead privacy engineer at Mozilla, told us that Mozilla is also recruiting privacy engineers. "We're looking for strong programmers who have a knack for understanding nuances of data sharing and use. They have an ability to build systems that enable transparency and can find ways to help our users make good choices through understanding risks of sharing their data," he said. However, Stamm said that he's had difficulty finding qualified candidates for privacy engineering positions: "I've found that technologists who find an interest in privacy get attracted to work in policy and activism. What we need are software engineers who want to write tools and features that will enhance people's privacy online."

\section{Training Privacy Engineers}

One way to address the privacy engineer shortage is to offer educational programs in the subject. During the past decade, several universities began offering privacyrelated courses in their computer science and engineering schools.

Our university, Carnegie Mellon, offers several privacy courses for undergraduate computer science and electrical and computer engineering majors; master's students in a variety of computer science, information systems, public policy, and business fields; and $\mathrm{PhD}$ students in computer science, engineering, and public policy. Privacyrelated courses include Information Security and Privacy; Privacy Policy, Law, and Technology; Usable Privacy and Security; Foundations of Security and Privacy; and Privacy in the Digital Age. Students who 
took all of these courses would have a solid foundation in privacy; however, typical students can't fit more than one or two of these courses into their schedule.

We've had a few students who focused their graduate coursework and research on privacy and have gone on to privacy-related careers. For example, one of our former $\mathrm{PhD}$ students went on to lead the World Wide Web Consortium's Do Not Track effort; another is a privacy manager at Microsoft. In addition, students who have gone on to other types of engineering roles in their careers have emailed us to report that they made use of their privacy expertise when their teams struggled with addressing privacy needs.

Over the past few years, we've been thinking about how to tie together our existing courses with some new privacy courses to create a comprehensive privacy engineering graduate curriculum. We've worked with our colleagues to propose an MS in Information Technology-Privacy Engineering (MSIT-PE) degree program and are currently recruiting students to enter the program next fall (http:// privacy.cs.cmu.edu).

MSIT-PE is a one-year program designed for computer scientists and engineers who want to pursue careers as privacy engineers or technical privacy managers. This program includes two semesters of courses taught by leading academic privacy and security experts. Students will take courses that cover legal and policy issues, the mathematical and technical foundations of privacy engineering, software engineering, usability assessment, and management as well as attend a weekly seminar covering current topics in privacy. The seminar will also feature guest lectures from privacy engineers working in the field. The program concludes with a summer-long capstone project in which students work as privacy consultants on client projects with students from other professional master's programs.

Currently, Carnegie Mellon is the only university to offer such a program, but we expect it won't be long before other universities offer similar degree programs. We've also observed a need for part-time and professional education programs for working professionals who want to gain privacy engineering expertise.

$\mathrm{P}$ rivacy engineering is emerging as a new career path that addresses the critical needs of business and government organizations. As Trevor Hughes, president and CEO of the IAPP, explained to us, "As the field of privacy grows around the globe, we are seeing a clear need for highly trained engineers who can translate the complexity of privacy into technology. There are too few of these professionals today." New courses and degree programs are needed to train students for these privacy engineering jobs.

\section{References}

1. A. Cavoukian, "Privacy by Design," Office of the Information and Privacy Commissioner, Aug. 2009; http: / / privacybydesign.ca/ publications/pbd-the-book.

2. K. Kindelan, "10 Privacy Blunders That 2010 Will Be Remembered For," SocialTimes, 21 Dec. 2010; http://socialtimes.com/10-privacy -blunders-that-2010-will-be -remembered-for_b32163.

3. Privacy Guidelines for Developing Software Products and Services, Microsoft, 29 Sept. 2010; www. microsoft.com/en-us/download/ details.aspx?id=16048.

4. S. Spiekermann and L.F. Cranor, "Engineering Privacy," IEEE Trans. Software Eng., vol. 35, no. 1, 2009, pp. 67-82.

5. "TRUSTe Unveils Top Online Privacy Predictions for 2012," TRUSTe, 19Dec. 2011; www.truste.
com/about_TRUSTe/press-room/ news_truste_top_privacy_predictions.

6. T. Romm, "Web Giants Tagged for Privacy Audits," Politico, 14 Dec. 2011; www.politico.com/news/ stories/1211/70453.html.

7. B. Bailey, "Google Recruiting Data Privacy 'Ninjas," Mercury News, 23 Aug. 2012; www.mercurynews. com/business/ci_21386267/ google-recruiting-data-privacy -ninja-red-team.

8. B. Gohring, "Google, Microsoft Teams Work to Keep Pace with Privacy Laws," ComputerWorld, 8 Dec. 2011; www.computerworld. com/s/article/9222536/Google _Microsoft_teams_work_to_keep _pace_with_privacy_laws.

Lorrie Faith Cranor is an associate professor in the School of Computer Science and in the Engineering \& Public Policy Department at Carnegie Mellon University. Contact her at lorrie@ cs.cmu.edu.

Norman Sadeh is a professor in the School of Computer Science at Carnegie Mellon University. Contact him atsadeh@cs.cmu.edu.

Selected CS articles and columns are also available for free at http://ComputingNow.computer.org.

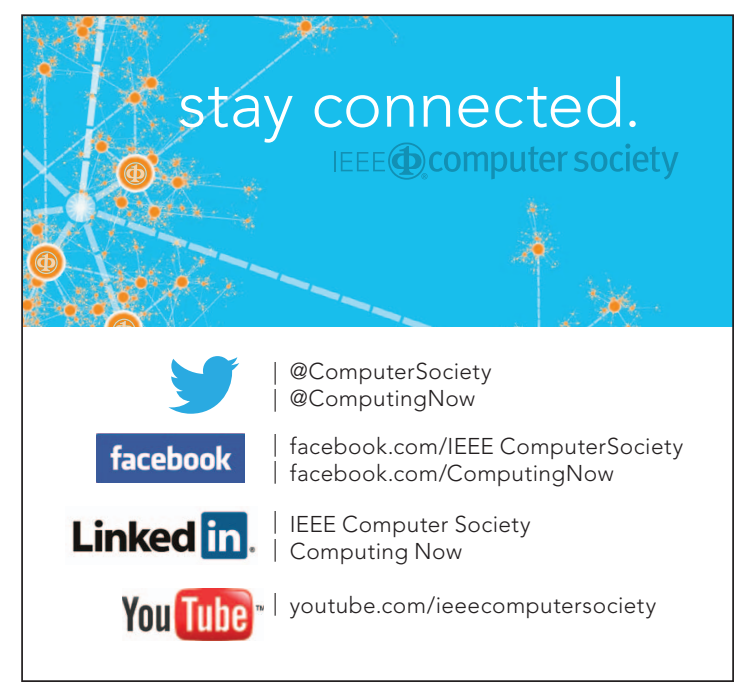

